\title{
Desenvolvimento de um aplicativo Android utilizando a classe FlingAnimation para abordagem de conceitos de cinemática
}

\author{
Development of an Android app using the FlingAnimation class to address kinematics concepts \\ Desarrollo de una aplicación de Android usando la clase FlingAnimation para acercar los conceptos \\ de cinemática
}

Recebido: 06/01/2021 | Revisado: 07/01/2021 | Aceito: 12/01/2021 | Publicado: 13/01/2021

\author{
Arilson José de Oliveira Júnior \\ ORCID: https://orcid.org/0000-0002-8508-1182 \\ Escola Técnica Estadual Dr. Domingos Minicucci Filho, Brasil \\ E-mail: arilsonjr@outlook.com \\ Rafael Plana Simões \\ ORCID: https://orcid.org/0000-0002-3433-8574 \\ Universidade Estadual Paulista "Júlio de Mesquita Filho", Brasil \\ E-mail: rafael.simoes@unesp.br
}

\begin{abstract}
Resumo
As dificuldades dos estudantes na aprendizagem de conceitos são atualmente desafios que os professores da disciplina de Física enfrentam durante o processo de ensino-aprendizagem. O uso de tecnologias como ferramenta didática é uma das formas adotadas para minimizar tais problemas. Entretanto, apesar dos resultados positivos que são obtidos com o uso dessas tecnologias no ensino de Física, ainda são poucos os aplicativos desenvolvidos para smartphones Android ${ }^{\mathbb{B}}$ com a finalidade de uso em sala de aula para abordagens de conceitos de Física. Assim, o presente trabalho foi realizado com o objetivo de desenvolver um aplicativo Android ${ }^{\circledR}$ utilizando a classe FlingAnimation para abordagens iniciais de conceitos de cinemática. Para o desenvolvimento utilizou-se a linguagem Java, por meio ambiente de desenvolvimento Android Studio. Na animação do objeto proposto no aplicativo, a classe FlingAnimation foi utilizada, sendo esta uma classe desenvolvida pelo Google para dar o suporte às animações em aplicativos. O aplicativo apresenta a trajetória retilínea de uma bola na simulação como um objeto puntiforme. Por fim, uma proposta didática é apresentada para que estudantes explorem o aplicativo de modo a conhecer suas funcionalidades e a compreender, inicialmente por uma percepção visual, como a variação do módulo da aceleração interfere no movimento de um corpo.
\end{abstract}

Palavras-chave: Cinemática; Android; Aplicativo; FlingAnimation; Dispositivos móveis.

\begin{abstract}
Students' difficulties in learning concepts are a currently challenge faced by Physics teachers during the teachinglearning process. The use of technologies as teaching tools is one of the ways adopted by teachers to minimize this kind of problem. However, despite the positive results that have been obtained by the use of these technologies in the Physics teaching, there are still few applications developed for Android ${ }^{\circledR}$ smartphones with the purpose of use in the classroom to address Physics concepts. Thus, the present study aimed the development of an Android ${ }^{\circledR}$ app using the FlingAnimation class for initial approaches of kinematics concepts. For the app development, the Java language was used through the Android Studio development environment. The class FlingAnimation was used for the animation of the object proposed in the app, which is a class developed by Google to support animations in apps. The app shows the straight trajectory of a ball in a simulation as a point object. Finally, a didactic proposal is presented to explore the app with students in order to elucidate its functionalities and to approach, initially through a visual perception, how the variation of the acceleration module interferes on the movement of a body.
\end{abstract}

Keywords: Kinematics; Android; App; FlingAnimation; Mobile devices.

\section{Resumen}

Las dificultades de los estudiantes para aprender conceptos son desafíos que enfrentan actualmente los profesores de física durante el proceso de enseñanza-aprendizaje. El uso de tecnologías como herramienta didáctica es una de las formas adoptadas para minimizar estos problemas. Sin embargo, a pesar de los resultados positivos que se obtienen con el uso de estas tecnologías en la enseñanza de la Física, aún son pocas las aplicaciones desarrolladas para smartphones Android ${ }^{\circledR}$ con el propósito de utilizarlas en el aula para aproximaciones a conceptos de Física. Así, el presente estudio tuvo como objetivo desarrollar una aplicación para Android ${ }^{\circledR}$, utilizando la clase FlingAnimation, para aproximaciones iniciales a los conceptos de cinemática. Para el desarrollo se utilizó el lenguaje Java, a través del entorno de desarrollo de Android Studio. En la animación del objeto propuesto en la aplicación se utilizó la clase FlingAnimation, que es una 
Research, Society and Development, v. 10, n. 1, e28410111710, 2021

(CC BY 4.0) | ISSN 2525-3409 | DOI: http://dx.doi.org/10.33448/rsd-v10i1.11710

clase desarrollada por Google para soportar las animaciones en aplicaciones. La aplicación presenta la trayectoria recta de una pelota en la simulación como un objeto puntual. Finalmente, se presenta una propuesta didáctica para que los estudiantes exploren la aplicación con el fin de conocer sus funcionalidades y comprender, inicialmente a través de una percepción visual, cómo la variación del módulo de aceleración interfiere con el movimiento de un cuerpo.

Palabras clave: Cinemática; Android; App; FlingAnimation; Dispositivos móviles.

\section{Introdução}

Dentre os principais problemas observados durante o processo de ensino-aprendizagem em aulas da disciplina de Física, as dificuldades dos estudantes em aprender conceitos, a falta de concentração e a desmotivação se constituem grandes desafios para os professores. Nesse sentido, diversas estratégias de ensino-aprendizagem têm sido propostas nas últimas décadas, das quais destaca-se o uso de tecnologias como ferramenta didática (Kielt, Silva \& Miquelin, 2017). Vários trabalhos apresentam situações de aprendizagem e propostas de planos de aula com uso de tecnologias para abordagem e facilitação da aprendizagem de conceitos de Física pelos estudantes. A grande parte desses trabalhos propõe o uso de simuladores desenvolvidos especificamente para ensino de ciências (Leal \& Oliveira, 2019; Silva, Sales \& Castro, 2018; Barbosa, et al., 2017; Fernandes, et al., 2016) ou a adaptação de aplicativos e acessórios para smartphones, como detectores de movimento, acelerômetros, niveladores, luxímetros, cartas celestes, realidade aumentada entre outros (Chiang \& Cheng, 2019; Díaz-Melián, 2019; Durelle, et al., 2017; Kielt, Silva \& Miquelin, 2017; Jesus \& Sasaki, 2016; Justiniano \& Botelho, 2016; Oliveira \& Manzano, 2016). Especificamente sobre o tema cinemática, alguns softwares e aplicativos foram desenvolvidos para auxiliar no processo de ensino-aprendizagem. Dentre eles destaca-se o Tracker (Silva \& Lima, 2019; Silva \& Sanabria, 2018; Brown \& Cox, 2009), um software desenvolvido a partir da iniciativa do projeto Open Source Physics (Osp, 2020). O programa é destinado à análise de vídeos de movimento quadro a quadro. Contudo, apesar dos resultados positivos que são obtidos com o uso dessas tecnologias no ensino de Física, ainda existem poucos aplicativos para smartphones especificamente desenvolvidos com a finalidade de uso em sala de aula para abordagens de conceitos de Física. Avaliando aplicativos para Android ${ }^{\circledR}$ disponíveis na plataforma Google Play, verifica-se a existência de aproximadamente 96 aplicativos desenvolvidos para abordagem de temas de Física. Considerando os aplicativos não pagos com alta popularidade - notas acima de 4,7 e instalação acima de 5000 dispositivos verifica-se a existência de apenas 11 aplicativos. Ainda assim, grande parte desses aplicativos disponíveis não se constituem como objeto educacional para o ensino de Física (como um simulador ou uma plataforma para aquisição de dados, por exemplo), mas como um conjunto de equações e/ou descrições de conceitos de Física. O uso dos recursos oferecidos pelos dispositivos móveis e outras tecnologias, como sensores e a própria computação móvel, permitem ao docente partilhar o conhecimento de maneira intuitiva, oferecendo ao estudante um meio pelo qual ele mesmo consiga observar e discutir um fenômeno, mediante um dispositivo que é seu alvo de atenção na maior parte do dia, os smartphones (Soga, Ueno-Guimarães \& Muramatsu, 2020; Orengo \& Schäffer, 2019; Jesus \& Sasaki, 2016).

Nesse sentido, o presente estudo apresenta o uso da classe FlingAnimation no desenvolvimento de um aplicativo Android $^{\circledR}$ para abordagem de conceitos de cinemática. Neste trabalho são apresentadas as implementações feitas para o desenvolvimento do aplicativo e suas relações com conceitos de cinemática.

\section{Metodologia}

\subsection{Desenvolvimento do aplicativo e suas relações com a cinemática}

A metodologia utilizada no desenvolvimento do trabalho foi de uma pesquisa experimental, baseada em observações diretas, predominantemente de natureza quantitativa (Pereira, et al., 2018). O aplicativo foi criado utilizando o ambiente de desenvolvimento Android Studio (Google, 2020b), por meio da linguagem de programação orientada a objetos Java (Oracle, 
Research, Society and Development, v. 10, n. 1, e28410111710, 2021

(CC BY 4.0) | ISSN 2525-3409 | DOI: http://dx.doi.org/10.33448/rsd-v10i1.11710

2020a). Adotou-se como versão alvo do aplicativo a API 28 (Android ${ }^{\circledR}$ Pie), sendo a versão mínima a API 15 (Android ${ }^{\circledR}$ Ice Cream Sandwich). A classe FlingAnimation foi desenvolvida na concepção de "movimento baseado em Física" (Physics-based Motion) que, segundo Google (2020a), está relacionada à ideia de que sempre que possível as animações em aplicativos/softwares devem aplicar conceitos da Física para ter uma aparência natural. Assim, a biblioteca de suporte do sistema operacional Android ${ }^{\circledR}$ inclui recursos de animação que obedecem às leis da Física (Google, 2020a). Atualmente há duas classes que trabalham com o conceito de movimento baseado em Física no pacote chamado androidx.dynamicanimation (Google, 2020d), sendo: SpringAnimation e FlingAnimation. Vale ressaltar que "uma classe é o projeto a partir do qual os objetos individuais são criados", e que "um objeto representa uma instância única de uma estrutura de dados definida pelo modelo fornecido por sua classe" (Oracle, 2020b). Portanto, FlingAnimation é uma classe desenvolvida pelo Google para dar suporte às animações que utilizam o conceito de "arremessar" um objeto (ou view) (Google, 2020c). Entende-se por objetos, nesse contexto, qualquer elemento visual dentro de um aplicativo, podendo ser um botão, uma tela, um ícone ou uma imagem qualquer.

Os métodos dessa classe dão o suporte necessário para aplicar dois tipos de movimentos: movimento com velocidade constante (valor mínimo de atrito) e movimento com velocidade variável (constante de atrito). Esses movimentos são condicionados de duas formas: definição da velocidade inicial do objeto pelo método "setStartVelocity()" e definição do valor de atrito (ou desaceleração) pelo método "setFriction()". Dessa forma, é possível aplicar o conceito de um movimento retilíneo uniformemente variável nas condições de um valor não nulo para o atrito (que no aplicativo faz com que a velocidade diminua de forma gradual). Por outro lado, definir o atrito como nulo (ou zero) torna o movimento com velocidade constante, ou seja, não havendo variação da velocidade ao longo do tempo: um movimento retilíneo uniforme. A codificação dos métodos que definem os diferentes tipos de movimento do objeto, teve como função principal a conversão das variações da posição do objeto - pixels ( $p x$ ) para milímetros (mm). Na Equação (1) é apresentada a função de conversão aplicada.

$$
s=\frac{254 s_{p x}}{d p i}
$$

Em que: $s$ é a posição do objeto (em mm), $s_{p x}$ é a posição do objeto (em pixel) e dpi é a densidade de pixels da tela do smartphone (em pixels por polegada), podendo ser: 160, 240, 320, 480 e 640.

Os dados de tempo são mostrados nas unidades de segundos e décimos de segundos. O aplicativo desenvolvido foi disponibilizado gratuitamente na plataforma Google Play com o nome BCinemática - MRU \& MRUV, para realizar o download basta acessar o endereço: https://play.google.com/store/apps/details?id=com.jrdev.ballmovement.

\subsection{Proposta de situação de aprendizagem utilizando o aplicativo}

Os conceitos iniciais no ensino de Física são comumente abordados de forma a compreender os fenômenos da natureza mais evidentes, como por exemplo, o movimento dos corpos. Após a compreensão de nomenclaturas e conceitos como: direção e sentido, grandeza Física escalar e vetorial, deslocamento, variação de espaço, ponto material e partícula; o estudo da Mecânica dá o início à construção de conhecimento sobre os fenômenos naturais mediante à Física (Mec, 2020). Dentro do tema Mecânica, a Cinemática é a responsável por abordar conceitos envolvidos no estudo dos movimentos dos corpos, desconsiderando suas causas e com o foco na descrição matemática desses movimentos (Nussenzveig, 1993).

Nesse sentido, o aplicativo apresenta a trajetória retilínea de uma bola tratada na simulação como um objeto puntiforme. O botão iniciar do aplicativo dá início ao movimento da bola com uma velocidade inicial de aproximadamente $\left|\overrightarrow{v_{0}}\right|=40 \mathrm{~mm} / \mathrm{s}$ (sendo este um valor considerado adequado para uma boa observação do movimento em uma tela de smartphone, tanto para MRU quanto para MRUV). O aplicativo apresenta também uma caixa de seleção onde o estudante pode selecionar quatro opções de desaceleração para o movimento da bola, sendo: $\left|\overrightarrow{a_{1}}\right|=0 \mathrm{~mm} / \mathrm{s}^{2} ;\left|\overrightarrow{a_{2}}\right|=10 \mathrm{~mm} / \mathrm{s}^{2} ;\left|\overrightarrow{a_{3}}\right|=20 \mathrm{~mm} / \mathrm{s}^{2}$ e; $\left|\overrightarrow{a_{4}}\right|=$ 
Research, Society and Development, v. 10, n. 1, e28410111710, 2021 (CC BY 4.0) | ISSN 2525-3409 | DOI: http://dx.doi.org/10.33448/rsd-v10i1.11710

$30 \mathrm{~mm} / \mathrm{s}^{2}$. Os valores predefinidos foram adotados de modo a permitir que o movimento possa ser visto adequadamente em todas as opções para uma velocidade inicial de $40 \mathrm{~mm} / \mathrm{s}$, considerando que valores maiores prejudicariam a percepção do movimento de desaceleração do objeto na tela. Ao escolher essas opções o estudante pode dar início à simulação de movimentação da bola em movimento retilíneo uniforme (para $\left|\overrightarrow{a_{1}}\right|=0 \mathrm{~mm} / \mathrm{s}^{2}$ ), ou movimento retilíneo uniformemente variado (para $\left|\overrightarrow{a_{2}}\right|=10 \mathrm{~mm} / \mathrm{s}^{2} ;\left|\overrightarrow{a_{3}}\right|=20 \mathrm{~mm} / \mathrm{s}^{2}$ e; $\left|\overrightarrow{a_{4}}\right|=30 \mathrm{~mm} / \mathrm{s}^{2}$ ). Devido ao fato de que a classe FlingAnimation apresenta apenas um método de definição do valor de atrito (ou desaceleração), o movimento está sempre associado a uma aceleração negativa, ou seja, o vetor aceleração é sempre oposto ao vetor velocidade, de forma que, quando $|\vec{a}| \neq 0$, o movimento sempre será retardado. A tela principal e as funções do aplicativo são apresentadas na Figura 1.

Figura 1. Tela principal do aplicativo BCinemática - MRU \& $M R U V$.

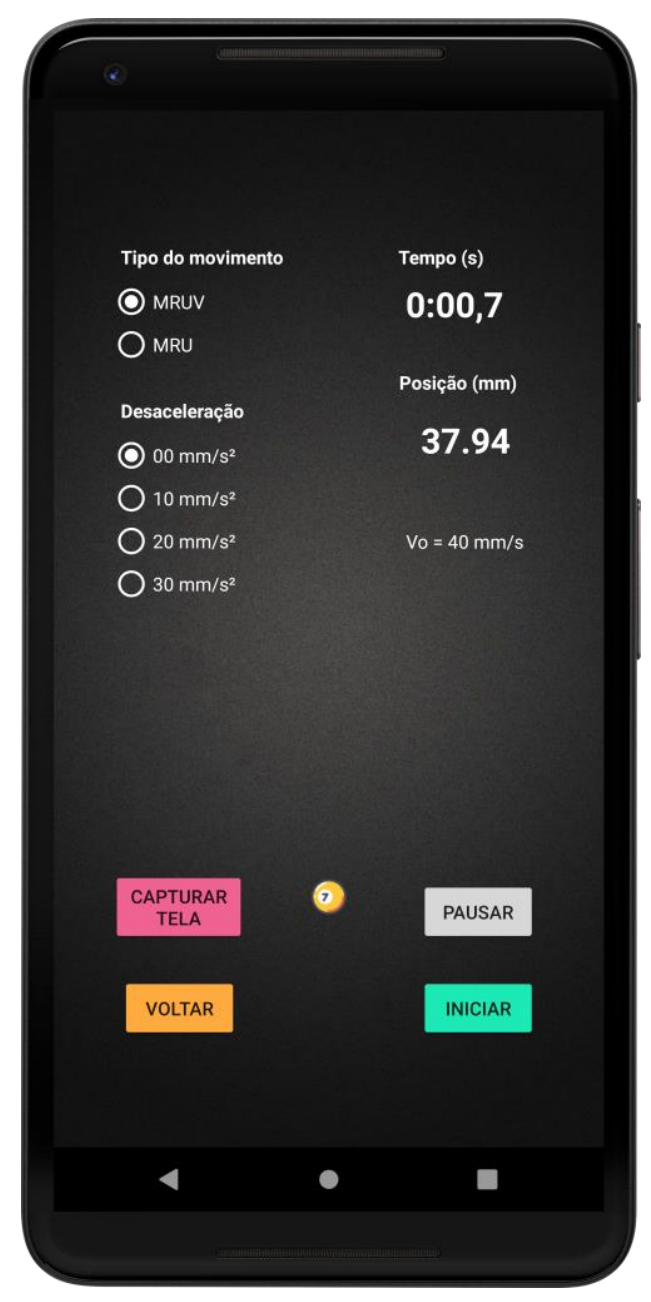

Fonte: Autores (2021).

A proposta é que os estudantes inicialmente instalem o aplicativo e o explorem de modo a conhecer suas funcionalidades e a compreender, inicialmente por uma percepção visual, como a variação do módulo da aceleração interfere no movimento de um corpo em movimento retilíneo uniforme ou movimento uniformemente variado (movimento retardado). $\mathrm{O}$ aplicativo possui também dois campos onde são apresentados os valores de variação de espaço $(\Delta s)$ e tempo $(t)$, os quais são atualizados a cada $0,1 s$ ao longo do deslocamento da bola. Com essa função, o docente pode solicitar que os estudantes façam a anotação desses valores para todas as opções de aceleração disponíveis, ou seja, para $\left|\overrightarrow{a_{1}}\right|=0 \mathrm{~mm} / \mathrm{s}^{2} ;\left|\overrightarrow{a_{2}}\right|=10 \mathrm{~mm} / \mathrm{s}^{2} ;\left|\overrightarrow{a_{3}}\right|=20 \mathrm{~mm} / \mathrm{s}^{2}$ e; $\left|\overrightarrow{a_{4}}\right|=30 \mathrm{~mm} / \mathrm{s}^{2}$. Além dos botões "Iniciar" e "Voltar", é permitido que o movimento da bola seja pausado, por meio do botão 
Research, Society and Development, v. 10, n. 1, e28410111710, 2021

(CC BY 4.0) | ISSN 2525-3409 | DOI: http://dx.doi.org/10.33448/rsd-v10i1.11710

"Pausar". Deste modo, os estudantes conseguem observar o deslocamento do objeto e seus respectivos valores de posição e tempo. Uma outra opção disponível no aplicativo para uma possível documentação durante a experimentação, é a opção de captura de tela (botão "Capturar tela"), no qual as imagens da tela do aplicativo são salvas no diretório público de downloads do dispositivo, no momento do clique do botão. Com isso, os estudantes poderão construir quatro tabelas de dados de espaço em função do tempo, sendo uma delas com dados de movimento retilíneo uniforme e três delas com movimento retilíneo uniformemente variado. O aplicativo, de forma intencional, não constrói essa tabela automaticamente. O objetivo dessa escolha é que os estudantes desenvolvam habilidades de aquisição e tabulação de dados obtidos de atividade experimental. Posteriormente à aquisição de dados, o professor pode solicitar aos estudantes que construam gráficos de espaço em função do tempo utilizando os dados obtidos com o uso do aplicativo. O objetivo dessa atividade é que o estudante perceba como é o comportamento da função $s(t)$ com a variação do módulo da aceleração.

\section{Resultados e Discussão}

\subsection{Proposta didática}

Ao executar as atividades propostas, o estudante irá obter uma tabela de dados de espaço em função do tempo, $s(t)$, para quatro valores diferentes de módulo de aceleração. Um exemplo de tabela de dados que poderá ser obtida é representa na Tabela 1. Novamente, deve-se salientar que esse conjunto de dados não é disponibilizado pelo aplicativo para que o estudante desenvolva habilidades de aquisição e tabulação de dados obtidos de atividade experimental. Essas habilidades são essenciais para o desenvolvimento de competências preconizadas pela Base Nacional Comum Curricular (Mec, 2020), em especial habilidades contempladas pela competência:

Investigar situações-problema e avaliar aplicações do conhecimento científico e tecnológico e suas implicações no mundo, utilizando procedimentos e linguagens próprios das Ciências da Natureza, para propor soluções que considerem demandas locais, regionais e/ou globais, e comunicar suas descobertas e conclusões a públicos variados, em diversos contextos e por meio de diferentes mídias e tecnologias digitais de informação e comunicação - TDIC (Mec, 2020).

Tabela 1. Exemplo de amostra de dados da função $s(t)$ que pode ser obtida pelo uso do aplicativo.

\begin{tabular}{|c|c|c|c|c|c|}
\hline Medida & $t(s)$ & $\begin{array}{c}s(\mathrm{~mm}) \text { para } \\
\left|\overrightarrow{a_{1}}\right|=00 \mathrm{~mm} / \mathrm{s}^{2}\end{array}$ & $\begin{array}{c}s(\mathrm{~mm}) \text { para } \\
\left|\overrightarrow{a_{2}}\right|=10 \mathrm{~mm} / \mathrm{s}^{2}\end{array}$ & $\begin{array}{c}s(\mathrm{~mm}) \text { para } \\
\left|\overrightarrow{a_{3}}\right|=20 \mathrm{~mm} / \mathrm{s}^{2}\end{array}$ & $\begin{array}{c}s(\mathrm{~mm}) \text { para } \\
\left|\overrightarrow{a_{4}}\right|=30 \mathrm{~mm} / \mathrm{s}^{2}\end{array}$ \\
\hline 1 & 0,1 & 13,18 & 13,18 & 13,18 & 13,18 \\
\hline 2 & 0,2 & 17,25 & 16,88 & 15,98 & 16,19 \\
\hline 3 & 0,3 & 22,28 & 20,48 & 19,26 & 19,05 \\
\hline 4 & 0,4 & 27,41 & 23,92 & 22,07 & 21,27 \\
\hline 5 & 0,5 & 32,49 & 27,09 & 24,45 & 23,02 \\
\hline 6 & 0,6 & 37,57 & 30,06 & 26,51 & 24,34 \\
\hline 7 & 0,7 & 42,60 & 32,86 & 28,20 & 25,40 \\
\hline 8 & 0,8 & 47,68 & 35,45 & 29,74 & 26,25 \\
\hline 9 & 0,9 & 52,76 & 37,84 & 30,96 & 26,83 \\
\hline
\end{tabular}

Fonte: Autores (2021).

Com os dados da Tabela 1 é possível construir um gráfico com as funções $s(t)$, sendo uma para cada valor diferente de módulo de aceleração. Nossa sugestão é que todo o conjunto de dados seja inserido em um único gráfico. Caso não seja possível, 
Research, Society and Development, v. 10, n. 1, e28410111710, 2021

é importante que os gráficos diferentes utilizem as mesmas escalas para os eixos $x$ e $y$. Essa sugestão tem como objetivo criar subsídios para que os estudantes notem que as curvas das funções $s(t)$ mudam de características, sendo uma reta para $\left|\overrightarrow{a_{1}}\right|=$ $0 \mathrm{~mm} / \mathrm{s}^{2}$ e funções quadráticas para $\left|\overrightarrow{a_{2}}\right|=10 \mathrm{~mm} / \mathrm{s}^{2} ;\left|\overrightarrow{a_{3}}\right|=20 \mathrm{~mm} / \mathrm{s}^{2}$ e; $\left|\overrightarrow{a_{4}}\right|=30 \mathrm{~mm} / \mathrm{s}^{2}$, com alteração das concavidades das parábolas para cada valor diferente da aceleração. A Figura 2 mostra um exemplo de gráfico que pode ser construído utilizando os dados obtidos com o desenvolvimento da situação de aprendizagem.

Figura 2. Exemplo de gráfico das funções $s(t)$ que podem ser construídos a partir dos resultados obtidos com a aplicação da situação de aprendizagem proposta.

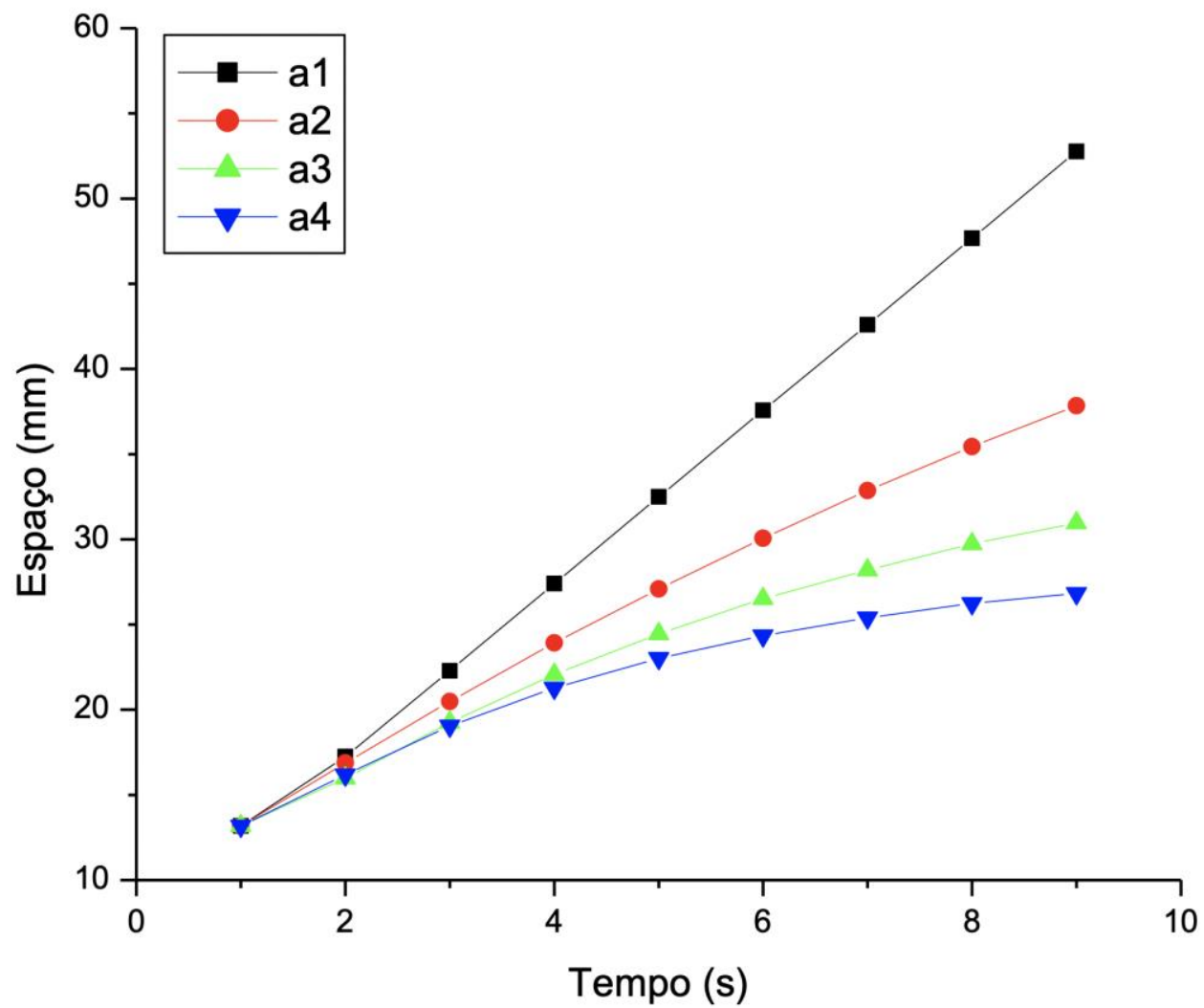

Fonte: Autores (2021).

Como forma de aprofundamento de conceitos, os estudantes podem utilizar os dados obtidos para determinação de funções de variação da velocidade em função do tempo $v(t)$. O módulo da velocidade para o tempo inicial (medida 1) é dado, sendo $\left|\overrightarrow{v_{1}}\right|=\frac{40 \mathrm{~mm}}{\mathrm{~s}}$. Já os demais valores podem ser obtidos utilizando a Equação 1. Considerando as medidas (coluna 1 da Tabela 1) como um índice $i$, esse cálculo pode ser simplesmente feito utilizando a Equação (2).

$$
\left|\overrightarrow{v_{l+1}}\right|=\frac{s_{i+1}-s_{i}}{t_{i+1}-t_{i}}
$$

A aplicação dos dados da Equação 2 sobre os dados da Tabela 1 irá gerar os resultados apresentados na Tabela 2 e os gráficos apresentados na Figura 3. 
Research, Society and Development, v. 10, n. 1, e28410111710, 2021

(CC BY 4.0) | ISSN 2525-3409 | DOI: http://dx.doi.org/10.33448/rsd-v10i1.11710

Tabela 2. Conjunto de dados da função $v(t)$ obtida a partir dos dados da Tabela 1.

\begin{tabular}{cccccc}
\hline Medida & $\boldsymbol{t}(\boldsymbol{s})$ & $\begin{array}{c}|\overrightarrow{\boldsymbol{v}}|(\mathbf{m m} / \mathbf{s}) \text { para } \\
\left|\overrightarrow{\boldsymbol{a}_{\mathbf{1}}}\right|=\mathbf{0 ~} \mathbf{m m} / \mathbf{s}^{\mathbf{2}}\end{array}$ & $\begin{array}{c}|\overrightarrow{\boldsymbol{v}}|(\mathbf{m m} / \mathbf{s}) \text { para } \\
\left|\overrightarrow{\boldsymbol{a}_{\mathbf{2}}}\right|=\mathbf{1 0} \mathbf{m m} / \mathbf{s}^{\mathbf{2}}\end{array}$ & $\begin{array}{c}|\overrightarrow{\boldsymbol{v}}|(\mathbf{m m} / \mathbf{s}) \text { para } \\
\left|\overrightarrow{\boldsymbol{a}_{3}}\right|=\mathbf{2 0} \mathbf{m m} / \mathbf{s}^{\mathbf{2}}\end{array}$ & $\begin{array}{c}|\overrightarrow{\boldsymbol{v}}|(\mathbf{m m} / \mathbf{s}) \text { para } \\
\left|\overrightarrow{\boldsymbol{a}_{\mathbf{4}}}\right|=\mathbf{3 0} \mathbf{m m} / \mathbf{s}^{\mathbf{2}}\end{array}$ \\
\hline 1 & 0,1 & 40 & 39 & 38 & 37 \\
2 & 0,2 & 40 & 38 & 36 & 34 \\
3 & 0,3 & 40 & 37 & 34 & 31 \\
4 & 0,4 & 40 & 36 & 32 & 28 \\
5 & 0,5 & 40 & 35 & 30 & 25 \\
6 & 0,6 & 40 & 34 & 28 & 22 \\
7 & 0,7 & 40 & 33 & 26 & 19 \\
8 & 0,8 & 40 & 32 & 24 & 16 \\
9 & 0,9 & 40 & 31 & 22 & 13 \\
\hline
\end{tabular}

Fonte: Autores (2021).

Figura 3. Exemplo de gráfico das funções $v(t)$ obtidas pela aplicação da Equação 1 sobre dos dados Tabela 1.

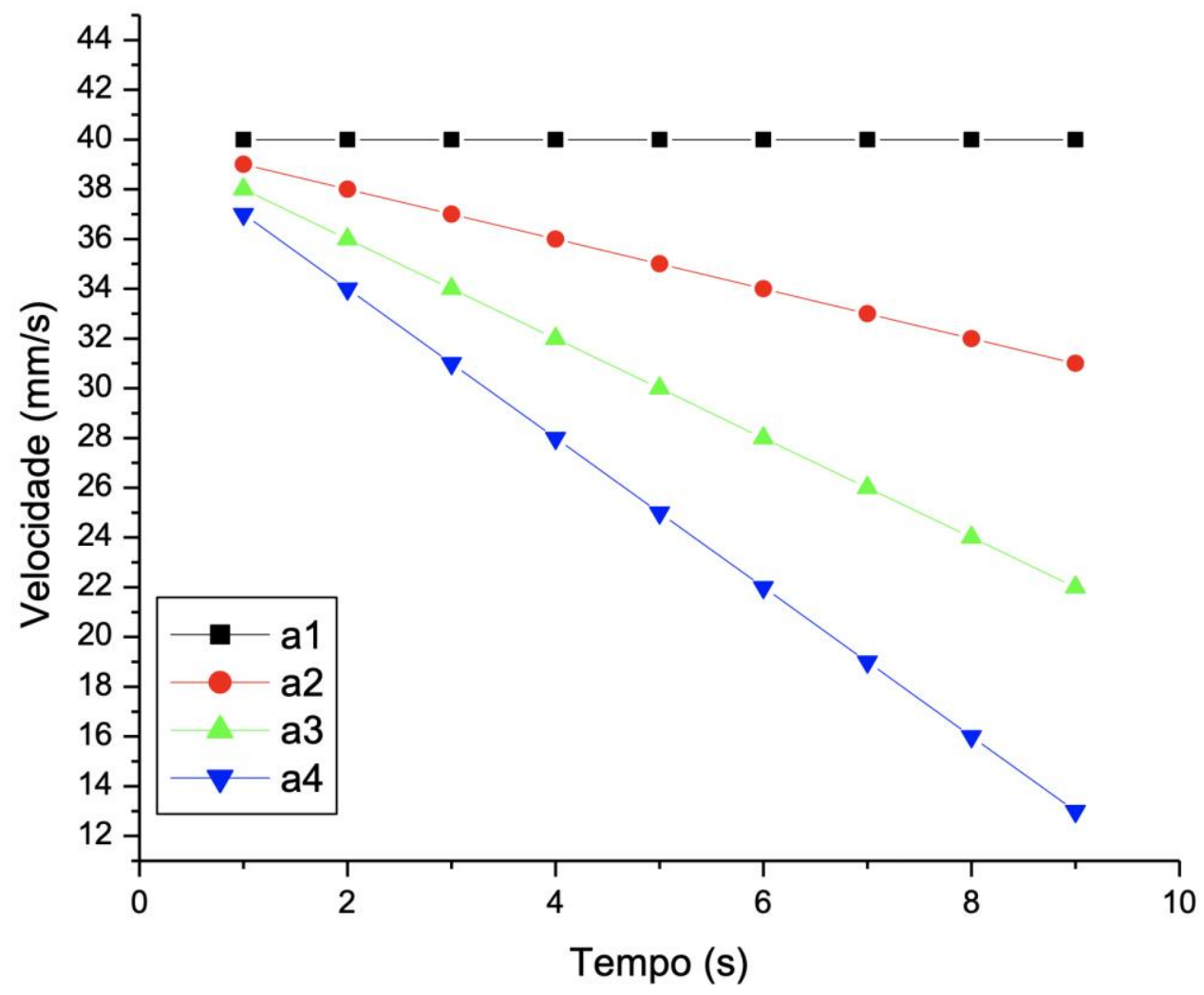

Fonte: Autores (2021).

A partir dos Gráficos ilustrados na Figura 3, conceitos sobre as características das funções $v(t)$ (ou seja, como a velocidade varia ao longo do tempo em um movimento retilíneo uniforme e em um movimento retilíneo uniformemente variado) poderão ser abordados. O professor poderá associar os dados dessas atividades às concepções prévias ou concepções espontâneas dos estudantes sobre velocidade que, especificamente para o ensino médio, são bastante intuitivas. Entretanto, é importante que, 
Research, Society and Development, v. 10, n. 1, e28410111710, 2021

(CC BY 4.0) | ISSN 2525-3409 | DOI: http://dx.doi.org/10.33448/rsd-v10i1.11710

nesse momento, a diferenciação entre os termos velocidade média e instantânea seja trabalhada, permitindo que fenômenos corriqueiros sejam avaliados em outras perspectivas, como por exemplo, a velocidade informada em um instante de tempo por um velocímetro de automóvel (Hewitt, 2002; Gualter \& Anré, 1997). Diferentemente dos conceitos de velocidade média e instantânea, o conceito de aceleração muitas vezes não é interpretado de forma correta pelos estudantes. Sendo assim, ao final da atividade os estudantes poderão determinar a aceleração média a partir dos resultados da Tabela 2 e verificar que os dados obtidos são iguais (ou aproximadamente iguais) às acelerações selecionadas no aplicativo para movimentar a bola.

\subsection{Aplicativo e classe FlingAnimation}

Inicialmente vale ressaltar que os dados de velocidade e aceleração presentes no aplicativo são aproximados, devido aos arredondamentos de valores como tempo, velocidade e aceleração, além de limitações da própria classe FlingAnimation. Conforme apresentado, essa classe foi desenvolvida a fim de permitir uma animação de lançamento para um objeto, podendo ser esse um botão, texto ou figura. Dessa forma, as definições de desaceleração dos objetos, por exemplo, são baseadas em um fator chamado de atrito (friction), que informa com que taxa ou razão a velocidade deve diminuir em uma animação. O valor atribuído a esse fator, mediante um método da classe, é um número real representado em ponto flutuante (float). Apesar de ser um fator que corresponde ao conceito de desaceleração, este não foi desenvolvido especificamente para se comportar como um valor de desaceleração. Podemos observar essa característica pelo fato de que não é permitido passar um valor nulo ou zero, por exemplo, mas sim um valor real próximo de zero.

Ao considerar o estudo do movimento de corpos, conceitos como tempo, deslocamento, velocidade e aceleração devem ser compreendidos. Entretanto, a classe FlingAnimation originalmente não dispõe de um método específico para determinação do valor de aceleração, apenas de alteração do fator de atrito e da velocidade inicial de um objeto. O que acaba por exigir uma implementação específica da função em uma possível modificação da classe. Todavia, observou-se que as limitações citadas não impedem o desenvolvimento das habilidades e competências propostas pela atividade. O movimento do objeto na tela do smartphone, bem como os efeitos da variação dos valores de desaceleração, não são prejudicados pelas aproximações e limitações da classe. Entende-se que as variações de espaço e tempo apresentadas na tela do dispositivo móvel, juntamente com o movimento do objeto, são suficientes para que o aluno consiga ter a percepção necessária das diferenças dos movimentos do tipo MRU e MRUV, ademais dos efeitos da desaceleração na bola. Ao comparar o BCinemática - MRU \& $M R U V$ com demais aplicativos disponíveis no Google Play, considerando os critérios de seleção citados no início desse trabalho, percebe-se que o aplicativo desenvolvido apresenta um carácter mais minimalista e objetivo quanto ao seu objeto de estudo, o que pode facilitar o aprendizado do aluno. Visando o uso do modelo adotado no presente trabalho por demais professores e pesquisadores, especialmente à aplicação da classe FlingAnimation em estudos envolvendo cinemática, o código-fonte do aplicativo é disponibilizado no repositório https://github.com/arjrr/bcinematicaapp. Espera-se que essa abordagem possa servir como fundamentação para desenvolvimento de novos softwares, assim como novas propostas de aprendizagem utilizando o aplicativo.

\section{Considerações Finais}

Os resultados obtidos mostraram que a classe FlingAnimation pode ser utilizada no desenvolvimento de aplicativos Android $^{\circledR}$ para a abordagem de conceitos de cinemática no ensino de Física. O uso do aplicativo BCinemática - MRU \& $M R U V$ demonstrou ser consistente ao estudo de movimento retilíneo uniforme e movimento retilíneo uniformemente variado e, dessa forma, apresentou potencial para utilização no ensino de Física como objeto educacional.

Considerando que a classe FlingAnimation não foi desenvolvida especificamente para abordagens de conceitos de cinemática, os métodos disponíveis na mesma são aceitáveis em aplicações pontuais, como as descritas no presente trabalho. 
Research, Society and Development, v. 10, n. 1, e28410111710, 2021 (CC BY 4.0) | ISSN 2525-3409 | DOI: http://dx.doi.org/10.33448/rsd-v10i1.11710

Todavia, faz-se necessário implementações que permitam observar outros comportamentos, como o de movimento acelerado. Trabalhos futuros devem ser desenvolvidos a fim de otimizar a classe, permitindo assim, que outros fenômenos físicos sejam estudados por alunos a partir de dispositivos móveis.

\section{Referências}

Barbosa, C. D., Gomes, L. M., Chagas, M. L., \& Ferreira, F. C. L. (2017). O uso de simuladores via smartphone no ensino de física: o experimento de oersted.: O experimento de Oersted. Scientia Plena, 13 (1), 1-13.

Brown, D., \& Cox, A. J. (2009). Innovative uses of video analysis. The Physics Teacher, 47 (1), 145-150.

Carvalho Neto, J. T., Apolinário, F. R., \& Soares, A. de A. (2017). Sistema photogate de seis canais analógicos para laboratórios didáticos de física. Revista Brasileira de Ensino de Física, 40 (1), e1504.

Chiang, C-M., \& Cheng H-Y. (2019). Use smartphones to measure Brewster's angle. The Physics Teacher, 57 (2), $118-119$.

Cid, A. S., \& Correa, T. (2019). Venturino: análise da variação de pressão em um tubo de Venturi utilizando Arduino e sensor de pressão. Revista Brasileira de Ensino de Física, 41 (3), e20180333.

Coelho, S. M., \& Séré, M. G. (1998). Pupils reasoning and practice during hands-on activities in the measurement phase. Research in Science \& Technological Edu- cation, Oxfordshire, 16 (1), 79-96.

Cordova, H. P., Aguiar, C. E., Amorim, H. S., Sathler, K. S. O. M., \& Santos, A. C. F. (2018). Audiotermômetro: um termômetro para a inclusão de estudantes com deficiência visual. Revista Brasileira de Ensino de Física, 40 (2), e2505.

Díaz-Melián, V. L., Rodríguez, L. A., Pedroso-Camejo, F., Mieres, J., De Armas, Y., Batista-Leyva, A. J., \& Altshuler, E. (2019) Optics Undergraduate Experiments Using Smart (and Not So Smart) Phones. Revista Cubana de Fisica, 36 (1), 4-7.

Durelle, J., Jones, J., Merriman, S., \& Balan, A. (2017). A smartphone-based introductory astronomy experiment: seasons investigation.: Seasons investigation. The Physics Teacher, 55 (2), 122-123.

Fernandes, A. C. P., Auler, L. T. S., Huguenin, J. A. O., \& Balthazar, W. F. (2016). Efeito Doppler com tablet e smartphone. Revista Brasileira de Ensino de Física, 38 (3), 1-8.

Guadagnini, P. H., Rocha, F. S., \& Barlette, V. E. (2019). Um medidor de luminosidade com módulo sensor integrado e aquisição automática de dados com aplicações didáticas. Revista Brasileira de Ensino de Física, 41 (3), e20180294.

Google. (2020a). Introduction to animations. https://developer.android.com/training/animation/overview\#physics-based.

Google. (2020b). Android Studio. https://developer.android.com/studio.

Google. (2020c). Move views using a fling animation. https://developer.android.com/guide/topics/graphics/fling-animation.

Google. (2020d). Dynamic animation. https://developer.android.com/reference/kotlin/androidx/dynamicanimation/animation/package-summary.

Jesus, V. L. B., \& Sasaki, D. G. G. (2016). Uma visão diferenciada sobre o ensino de forças impulsivas usando um smartphone. Revista Brasileira de Ensino de Física, 38 (1), 1-6.

Justiniano, A., \& Botelho, R. (2016). Construção de uma carta celeste: um recurso didático para o ensino de astronomia nas aulas de física: Um recurso didático para o ensino de Astronomia nas aulas de Física. Revista Brasileira de Ensino de Física, 38 (4), 1-11.

Kielt, E. D., Silva, S. C. R., \& Miquelin, A. F. (2017). Implementação de um aplicativo para smartphones como sistema de votação em aulas de Física com Peer Instruction. Revista Brasileira de Ensino de Física, 39 (4), 1-8.

Leal, T. C. S., \& Oliveira, A. A. (2019). Utilização de plataformas interativas e novas tecnologias no ensino de física das radiações para cursos da área de saúde. Revista Brasileira de Ensino de Física, 41 (4), 1-7.

Mec. (2020). Base Nacional Comum Curricular. http://basenacionalcomum.mec.gov.br/a-base.

Nascimento Júnior, J. F., Borges, V. E. S., \& Nascimento, R. M. M. F. (2019). Descrição temporal de forças de colisão: um modelo didático para laboratório de física assistido por sistema embarcado. Revista Brasileira de Ensino de Física, 41 (3), e20180219.

Nussenzveig, H. M. (1993). Curso de Física básica: EdgardBlucher.

Oliveira, L. D., \& Manzano, R. C. Aplicações de realidade aumentada no ensino de Física a partir do software LAYAR. Renote, 14 (1), 1-10.

Osp. (2020). Open Source Physics. http://www.compadre.org/osp/.

Oracle. (2020a). Java. https://www.oracle.com/java/. 
Research, Society and Development, v. 10, n. 1, e28410111710, 2021 (CC BY 4.0) | ISSN 2525-3409 | DOI: http://dx.doi.org/10.33448/rsd-v10i1.11710

Oracle. (2020b). Classes and Objects. https://docs.oracle.com/cd/E91187_01/pt855pbr2/eng/pt/tpcd/concept_ClassesandObjects074b14.html?pli=ul_d61e48_tpcd.

Orengo, G., \& Schäffer, D. (2020). Os dados nucleares da Agência Internacional de Energia Atômica (IAEA) como aporte científico no Ensino de Física Nuclear. Revista Brasileira de Ensino de Física, 42 (1), 1-7.

Pereira, A. S., Shitsuka, D. M., Parreira, F. J., \& Shitsuka, R. (2018). Metodologia da pesquisa científica. RS: UFSM, NTE.

Séré, M., Coelho, S. M., \& Nunes, A. D. (2003). O papel do ensino da física experimentação no. Cad.Bras.Ens.Fís., 20 (1), $30-42$.

Silva, E. S., \& Lima, A. R. (2019). Estimando o coeficiente de atrito cinético entre duas superfícies por meio da vide análise. Revista Brasileira de Física Tecnológica Aplicada, 6 (1), 14-27.

Silva, D., Sales, G., \& Castro, J. (2018). A utilização do aplicativo plickers como ferramenta na implementação da metodologia peer instruction. Revista Eletrônica Científica Ensino Interdisciplinar, 4 (12), 502-516.

Silveira, M. V., Barthem, R. B., \& Santos, A. C. (2018). Proposta didático experimental para o ensino inclusivo de ondas no ensino médio. Revista Brasileira de Ensino de Física, 41 (1), e20180084.

Silva, E. S., \& Sanabria, N. D. (2018). Video análise de disparos realizados por uma catapulta caseira: uma proposta de ensino para a discussão de lançamentos oblíquos e avaliação da energia mecânica. Revista Brasileira de Física Tecnológica Aplicada, Ponta Grossa, 5 (1), 14-26.

Soga, D., Ueno-Guimarães, M. H., \& Muramatsu, M. (2020). Um Estudo Experimental sobre a Luz Negra com Smartphone. Revista Brasileira de Ensino de Física, $42(1), 1-5$. 